\title{
Correlación entre criterios clínicos y de laboratorio de casos notificados por sospecha de hantavirosis y el resultado de la técnica de referencia
}

\author{
Maritza Navarrete, Melissa Hott, Jorge Caroca, Luis Leyton, Nadialett Venegas, \\ Kissy Ismail, Farides Saavedra y Carola Otth
}

\section{Correlation between clinical and laboratory criteria of suspected Hantavirus cases and the results of the reference diagnostic technique}

Background: Currently in Chile, due to the frequent clinical suspicion of Hantavirus disease and the high public health impact that this causes, it is necessary to strengthen the criteria for clinical and epidemiological suspicion in the health team. Objective: To analyze the information contained in the reports of suspected Hantavirus infection versus the confirmatory diagnosis with the reference technique, IgM capture ELISA anti-hantavirus. Material and Methods: Correlation between the information provided in notifications versus the result of confirmation was analyzed by calculating diagnostic accuracy. Results: $3.4 \%$ of 1,566 patients studied (53 cases) was confirmed as SCPH. $58.6 \%$ of the analyzed notifications was incomplete. The percentage of positivity of the reference technique associated with fever, myalgia and headache was $80-85 \%$. The presence of immunoblasts $(>10 \%)$ showed $25 \%$ sensitivity, 98\% specificity, 37\% PPV, 97\% NPV. Thrombocytopenia exhibited 98\% sensitivity, 74\% specificity, $16 \% \mathrm{PPV}, 100 \% \mathrm{NPV}$. Conclusion: It is necessary to reinforce the importance of comprehensive data reporting at the health system level. The presence of thrombocytopenia and immunoblasts $(>10 \%)$ is highly sensitive and specific, respectively, for detecting patients with SCPH. There is a need to develop training programs in order to optimize the suspicion of Hantavirus infection and appropriate use of health resources.

Key words: Hantavirus, hantavirus cardiopulmonary syndrome, diagnostic, POC-PUU, Andesvirus.

Palabras clave: Hantavirus, síndrome cardiopulmonar por hantavirus, diagnóstico, POC-PUU, Andesvirus.
Universidad Austral de Chile, Valdivia, Chile. Facultad de Medicina. Instituto de Microbiología Clínica (MN, MH, JC, LL, FS, CO). Hospital Base Valdivia, Valdivia, Chile. Unidad de Microbiología, Infectología y Farmacología Clínica (MN, MH, LL)

Secretaría Regional Ministerio de Salud (SEREMI), Región de la Araucanía, Chile (NV, KI).

Financiamiento: Proyecto FICR-20, DID-UACH, Convenio Hantavirus SSV-UACH

Los autores declaran no tener conflicto de intereses.

Recibido: 2 de noviembre de 2015 Aceptado: 14 de abril de 2016

Correspondencia a: Carola Otth Lagunas cotth@uach.cl

\section{Introducción}

$\mathrm{E}$ n Chile, desde mediados de la década del 90 se han notificado casos del síndrome cardiopulmonar por hantavirus ( $\mathrm{SCPH}$ ), aunque estudios serológicos retrospectivos han demostrado la presencia de anticuerpos contra hantavirus en muestras de pacientes desde el año $1975^{1,2}$.

El agente etiológico del SCPH en nuestro país es un virus ARN del género Hantavirus, familia Bunyaviridae, especie Andes (ANDV) ${ }^{3}$. Su reservorio principal es el "ratón de cola larga" (Oligoryzomys longicaudatus), el cual se distribuye desde la Región de Atacama hasta la Región de Magallanes ${ }^{4}$. El mecanismo de transmisión de ANDV a los humanos es a través de la inhalación de aerosoles conteniendo partículas virales provenientes de la orina o saliva de roedores infectados o bien, por el contacto directo con sus excretas ${ }^{5-7}$.

La infección es endémica, con brotes estacionales que se inician a fines de primavera, presentándose la mayoría de los casos entre los meses de noviembre y abril. Han sido notificados casos de sospecha de infección por hantavirus desde la Región de Valparaíso hasta la Región de Aysén (entre $32^{\circ}$ y $48^{\circ}$ latitud sur), distribuyéndose la mayoría de ellos entre las regiones del Biobío y de Aysén ${ }^{8}$.

En nuestro país, se han confirmado 963 casos de enfermedad por hantavirus (1993-2015) donde se incluyen pacientes con manifestaciones clínicas leves y graves (http://epi.minsal.cl/wp-content/uploads/2016/03/ Informe-semanal-hantavirus-se112016.pdf).

La infección por ANDV produce un cuadro clínico que se caracteriza por fiebre asociada a síntomas inespecíficos como cefalea, mialgias, escalofríos, malestar general, síntomas gastrointestinales (fase prodrómica); seguidos por una súbita insuficiencia respiratoria e inestabilidad hemodinámica (fase cardiopulmonar) que, en algunos casos, puede evolucionar hacia un distress respiratorio, con shock, falla orgánica múltiple (FOM) y muerte ${ }^{8}$. Adicionalmente, en el contexto de la FOM, el virus puede producir compromiso de otros sistemas, evolucionando con coagulopatía, insuficiencia renal, hepatitis y pancreatitis, entre otras ${ }^{8}$. 
La sospecha clínica de enfermedad por hantavirus en su etapa prodrómica debe plantearse teniendo en cuenta: antecedentes epidemiológicos de factores de riesgo (labores agro-forestales, residentes en sectores rurales, excursionistas en sectores rurales); la presencia de fiebre y mialgias con o sin síntomas gastrointestinales; leucocitosis $\left(>12.000 / \mathrm{mm}^{3}\right)$, presencia de inmunoblastos (linfocitos atípicos) $>10 \%$, hemoconcentración y plaquetas $<150.000 / \mathrm{mm}^{3}$; la radiografía de tórax, inicialmente puede ser normal, pero lo característico es un patrón intersticial uni o bilateral ${ }^{9}$.

La confirmación diagnóstica de hantavirus se efectúa mediante la detección de anticuerpos (IgM e IgG) por medio de la técnica de ELISA (técnica considerada el estándar de oro) y/o detección del ARN viral mediante RPC-RT (reacción de polimerasa en cadena acoplada a retro-transcripción $)^{9}$. En Chile, el diagnóstico se realiza en el Instituto de Salud Pública de Chile (ISP), centro de referencia nacional, coordinadamente con dos centros de confirmación: la Universidad Austral de Chile en Valdivia (UACH), para las regiones: Araucanía, Los Lagos y Los Ríos, y la Pontificia Universidad Católica de Chile (PUC). A partir de febrero de 2012 el ISP estableció la red de vigilancia de laboratorios públicos para hantavirus desde la Región de Valparaíso a la Región de los Lagos, con el objetivo de cubrir las necesidades de un diagnóstico precoz de esta enfermedad mediante la aplicación de pruebas rápidas comerciales: test inmunocromatográfico POCPUU con antígenos heterólogos de hantavirus PUUV y ELISA Pool América 2, con antígenos: virus Sin Nombre (SNV) y ANDV ${ }^{9}$. Dichas pruebas sirven como tamizaje inicial de apoyo, siendo necesario el envío inmediato de las muestras para una confirmación diagnóstica por los centros de referencias.

Al realizar un análisis preliminar de nuestra labor como laboratorio de confirmación, destaca la gran cantidad de casos de sospecha de enfermedad por hantavirus que finalmente son negativos. Dada la situación de elevada sospecha clínica que finalmente son casos en los cuales se descarta la enfermedad, y el alto impacto en la salud pública que involucra un mayor gasto de exámenes y días cama, se hace necesario reforzar los criterios de sospecha clínicos y epidemiológicos al equipo de salud, con el fin de optimizar la sospecha y el uso adecuado de los recursos sanitarios.

Los objetivos del presente trabajo fueron:

- Establecer el grado de adherencia y cumplimiento del registro de datos requerido en el formulario de notificación; y

- Analizar la correlación de los datos registrados (criterios clínicos y de laboratorio) respecto a la serología confirmatoria por la técnica considerada estándar de oro: ELISA IgM de captura anti-hantavirus.

\section{Materiales y Métodos}

\section{Datos asociados a las notificaciones de sospecha de infección por hantavirus}

Fueron analizados los antecedentes clínicos y las características del hemograma de 1.566 notificaciones de sospecha de infección por hantavirus recibidas en el Laboratorio de Virología Molecular de la UACH, entre febrero de 2012 y julio de 2014, provenientes de pacientes de las regiones del Sur de Chile: Araucanía, Los Ríos y los Lagos. Dichas notificaciones se realizan al momento de la consulta inicial del paciente y derivación de la muestra a los laboratorios de referencia.

\section{Algoritmo diagnóstico}

En las regiones antes mencionadas, las muestras de pacientes con sospecha de infección por hantavirus son analizadas en los laboratorios públicos definidos para la red de vigilancia, mediante pruebas serológicas de tamizaje rápido usando los kits POC-PUU o ELISA POOL 2 (validados por ISP para su uso) ${ }^{9}$. Independientemente del resultado de estas pruebas rápidas las muestras deben ser transportadas bajo medidas de bioseguridad estándar al Laboratorio de Virología Molecular del Instituto de Microbiología Clínica de la UACH Chile, donde son analizadas mediante la técnica de referencia ELISA IgM de captura anti-hantavirus con antígenos del virus Laguna Negra (LNV) o ANDV y ELISA indirecto IgG antiANDV. Este método consiste en la detección serológica de anticuerpos $\operatorname{IgM}$ e IgG anti-hantavirus mediante un ensayo inmunoenzimático (ELISA) utilizando proteína recombinante de la nucleocápside de $\mathrm{ANDV}^{10,11} \mathrm{o}$ de LNV (que presenta reacción cruzada con otros hantavirus como ANDV). Para IgM anti-hantavirus se utiliza la técnica ELISA IgM de captura, y para detectar IgG anti-hantavirus se utiliza la técnica indirecta de ELISA IgG. Los resultados son informados dentro de las $24 \mathrm{~h}$ de recibida la muestra en el laboratorio.

\section{Análisis de los datos}

Se analizó la precisión diagnóstica de los parámetros trombocitopenia $\left(<150.000\right.$ plaquetas $\left./ \mathrm{mm}^{3}\right)$, presencia de inmunoblastos $(>10 \%)$ y hemoconcentración (según parámetros establecidos como valores normales por edad y sexo, como valores de referencia de los laboratorios clínicos de los hospitales de origen de los casos sospechosos), y los parámetros clínicos de pacientes con sospecha de infección por hantavirus, consignados en la ficha de notificación ${ }^{9}$ en comparación con la técnica de confirmación o referencia. Se calculó la sensibilidad (S), especificidad (E), valor predictor positivo (VPP), valor predictor negativo (VPN) y cociente de probabilidad -LR (en inglés likehood ratio), de acuerdo a lo descrito en la Tabla 1. 


\begin{tabular}{|c|c|c|}
\hline Parámetro & Fórmula de Cálculo & Definición \\
\hline Sensibilidad & $\begin{array}{l}\mathrm{S}=100 \times(\text { concordancia de positivos del parámetro analizado y estándar } \\
\text { de oro }) /(\text { concordancia de positivos del parámetro analizado y estándar } \\
\text { de oro }+ \text { falsos negativos por parámetro analizado) }\end{array}$ & $\begin{array}{l}\text { Proporción de individuos con la enfermedad que tienen una prueba } \\
\text { positiva }\end{array}$ \\
\hline Especificidad & $\begin{array}{l}\mathrm{E}=100 \times(\text { concordancia de negativos del parámetro analizado y estándar } \\
\text { de oro }) /(\text { concordancia de negativos del parámetro analizado y estándar } \\
\text { de oro }+ \text { falsos positivos por parámetro analizado) }\end{array}$ & Proporción de sanos que tienen una prueba negativa \\
\hline $\begin{array}{l}\text { Valor predictor } \\
\text { positivo }\end{array}$ & $\begin{array}{l}\text { VPP }=100 \times(\text { concordancia de positivos del parámetro analizado y } \\
\text { estándar de oro) } / \text { (concordancia de positivos del parámetro analizado y } \\
\text { estándar de oro }+ \text { falsos positivos por parámetro analizado). }\end{array}$ & $\begin{array}{l}\text { Probabilidad de que un individuo con resultado positivo en la prueba, } \\
\text { tenga la enfermedad }\end{array}$ \\
\hline $\begin{array}{l}\text { Valor predictor } \\
\text { negativo }\end{array}$ & $\begin{array}{l}\text { VPN }=100 \times \text { (concordancia de negativos del parámetro analizado y } \\
\text { estándar de oro) } / \text { (concordancia de negativos del parámetro analizado y } \\
\text { estándar de oro }+ \text { falsos negativos por parámetro analizado) }\end{array}$ & $\begin{array}{l}\text { Probabilidad de que un individuo con resultado negativo en la prueba, no } \\
\text { tenga la enfermedad }\end{array}$ \\
\hline $\begin{array}{l}\text { Likelihood ratio } \\
\text { positivo }\end{array}$ & LR+ = Sensibilidad / (1-Especificidad) & $\begin{array}{l}\text { Proporción de enfermos que han tenido un resultado positivo en la prueba } \\
\text { diagnóstica dividido entre la proporción de sanos que también han dado } \\
\text { positivo en dicha prueba }\end{array}$ \\
\hline $\begin{array}{l}\text { Likelihood ratio } \\
\text { negativo }\end{array}$ & LR- = (1-Sensibilidad)/Especificidad & $\begin{array}{l}\text { Proporción de enfermos que han dado un resultado negativo en la prueba } \\
\text { diagnóstica dividido entre la proporción de sanos que también han dado } \\
\text { negativo en dicha prueba }\end{array}$ \\
\hline
\end{tabular}

\begin{tabular}{|c|c|c|c|c|c|c|c|c|c|}
\hline $\begin{array}{l}\text { Informe de } \\
\text { síntoma }\end{array}$ & Fiebre & Cefalea & Mialgia & $\begin{array}{c}\text { Síndrome } \\
\text { gastrointestinal }\end{array}$ & $\begin{array}{l}\text { Compromiso } \\
\text { respiratorio }\end{array}$ & $\begin{array}{l}\text { Infiltrado } \\
\text { de tórax }\end{array}$ & Inmunoblastos & Hemoconcentración & Trombocitopenia \\
\hline Presente & $\begin{array}{c}1.247 \\
(79,6 \%)\end{array}$ & $\begin{array}{c}1.176 \\
(75,1 \%)\end{array}$ & $\begin{array}{l}1.128 \\
(72 \%)\end{array}$ & $\begin{array}{c}837 \\
(53,4 \%)\end{array}$ & $\begin{array}{c}412 \\
(26,3 \%)\end{array}$ & $\begin{array}{c}287 \\
(18,3 \%)\end{array}$ & $\begin{array}{c}19 \\
(1,2 \%)\end{array}$ & $\begin{array}{c}87 \\
(5,6 \%)\end{array}$ & $\begin{array}{c}252 \\
(16,1 \%)\end{array}$ \\
\hline Ausente & $\begin{array}{c}227 \\
(14,5)\end{array}$ & $\begin{array}{c}251 \\
(16 \%)\end{array}$ & $\begin{array}{c}277 \\
(17,7 \%)\end{array}$ & $\begin{array}{c}551 \\
(35,2 \%)\end{array}$ & $\begin{array}{c}877 \\
(56 \%)\end{array}$ & $\begin{array}{c}401 \\
(25,6 \%)\end{array}$ & $\begin{array}{c}630 \\
(40,2 \%)\end{array}$ & $\begin{array}{c}734 \\
(46,9 \%)\end{array}$ & $\begin{array}{c}603 \\
(38,5 \%)\end{array}$ \\
\hline No informado & $92(5,9 \%)$ & $\begin{array}{c}139 \\
(8,9 \%)\end{array}$ & $\begin{array}{c}161 \\
(10,3 \%)\end{array}$ & $\begin{array}{c}178 \\
(11,4 \%)\end{array}$ & $\begin{array}{c}277 \\
(17,7 \%)\end{array}$ & $\begin{array}{c}878 \\
(56,1 \%)\end{array}$ & $\begin{array}{c}917 \\
(58,6 \%)\end{array}$ & $\begin{array}{c}745 \\
(47,5 \%)\end{array}$ & $\begin{array}{c}711 \\
(45,4 \%)\end{array}$ \\
\hline Total & $\begin{array}{c}1566 \\
(100 \%)\end{array}$ & $\begin{array}{c}1.566 \\
(100 \%)\end{array}$ & $\begin{array}{c}1.566 \\
(100 \%)\end{array}$ & $\begin{array}{c}1.566 \\
(100 \%)\end{array}$ & $\begin{array}{c}1.566 \\
(100 \%)\end{array}$ & $\begin{array}{c}1.566 \\
(100 \%)\end{array}$ & $\begin{array}{c}1.566 \\
(100 \%)\end{array}$ & $\begin{array}{c}1.566 \\
(100 \%)\end{array}$ & $\begin{array}{c}1.566 \\
(100 \%)\end{array}$ \\
\hline
\end{tabular}

\section{Resultados}

Entre febrero de 2012 y agosto de 2014, el Laboratorio de Virología Molecular de la UACH, recibió un total de 1.566 muestras con sospecha de infección por hantavirus procedentes de las regiones La Araucanía, Los Ríos y Los Lagos, las que contaban con sus correspondientes formularios de notificación. Del análisis de los datos demográficos entregados en dichos formularios, se pudo establecer que el promedio de edad fue de 33 años (rango $1-96$ años), con $63,9 \%$ de hombres (1.001 casos) y $36,0 \%$ mujeres (565 casos). De las 1.566 muestras recibidas por sospecha de infección por hantavirus, 3,4\% (53 casos) fueron positivas por la técnica de confirmación. Todas las notificaciones por casos de sospecha de infección por hantavirus recibidas en el laboratorio consignaban algún factor de riesgo epidemiológico, tales como labores agro-forestales, residentes en sectores rurales o excursionistas en sectores rurales. De las 1.566 notificaciones de casos sospechosos, sólo $41,4 \%$ consignaba el total de la información requerida por el Departamento de Epidemiología del Ministerio de Salud. De los síntomas y signos prodrómicos consignados en la notificación como presente o ausente, figuran con mayor porcentaje de registro los datos de fiebre, cefalea, mialgias y síntomas gastrointestinales. Se observó un alto porcentaje de datos no informados o no registrados en la notificación, para los parámetros imagenológicos (radiografía de tórax) 56,1\%, y de laboratorio entre 45,4-56,8\% (Tabla 2).

Para cada parámetro del hemograma (trombocitopenia, 
Tabla 3. Relación entre presencia y ausencia de los parámetros de laboratorio: trombocitopenia (A), inmunoblastos (B) y hemoconcentración (C) en relación a serología IgM anti-hantavirus por método de referencia

$\begin{array}{lccc}\text { A } & \text { Trombocitopenia } \\ \text { Serología } & \begin{array}{c}\text { Trombocitopenia } \\ \text { Negativa }\end{array} & \text { Total } \\ \text { hantavirus } & 40 & 1 & 41 \\ \text { IgM Positiva } & 212 & 602 & 814 \\ \text { IgM Negativa } & 252 & 603 & 855 \\ \text { Total } & & \end{array}$

S: $98 \% ;$ E: 74\%; VPP: 16\%; VPN: 100\%; LR+: 3,75; LR-: 0,03.

B

\begin{tabular}{lccc}
$\begin{array}{l}\text { Serología } \\
\text { hantavirus }\end{array}$ & $\begin{array}{c}\text { Inmunoblastos } \\
\text { Positivos }\end{array}$ & $\begin{array}{c}\text { Inmunoblastos } \\
\text { Negativo }\end{array}$ & Total \\
IgM Positiva & 7 & 21 & 28 \\
IgM Negativa & 12 & 609 & 621 \\
Total & 19 & 630 & 649 \\
\hline
\end{tabular}

S: 25\%; E: 98\%; VPP: 37\%; VPN: 97\%; LR+: 12,94; LR-: 0,76.

C

Serología hantavirus

IgM Positiva

IgM Negativa

Total

S: 43\%; E: 91\%; VPP: 18\%; VPN: 97\%; LR+: 4,78; LR-: 0,62 inmunoblastos $>10 \%$, hemoconcentración) y signos clínicos (fiebre, cefalea, compromiso respiratorio), se determinó la precisión diagnóstica de sospecha de infección por hantavirus en relación a ELISA IgM de captura, calculando: S, E, VPP, VPN, LR- y LR+ (Tablas 3 y 4), de acuerdo a las fórmulas descritas en la Tabla 1.

Las Tablas 3A, 3B, 3C muestran que el parámetro menos sensible pero más específico fue la presencia de inmunoblastos $(>10 \%)$, con una $\mathrm{S}$ de $25 \%$ y E de $98 \%$. El criterio más sensible fue la trombocitopenia con $98 \%$, pero con una $\mathrm{E}$ de $74 \%$, mientras que la hemoconcentración presentó S de 43\% y E de 91\%. La presencia de inmunoblastos (> 10\%) presenta el VPP más alto (37\%). Todos los criterios del hemograma consignados en la notificación de sospecha de $\mathrm{SCPH}$, presentaron un VPN $>90 \%$ (rango 97-100\%).

En relación a los signos clínicos (Tabla 4A, 4B, 4C) destaca el parámetro fiebre con $\mathrm{S}$ de $90 \%$ y VPN de $98 \%$; para el compromiso respiratorio se observa una baja $\mathrm{S}$ de $50 \%$.

\section{Discusión}

La enfermedad causada por hantavirus (SCPH) en Chile ha evolucionado como un problema emergente de salud pública, presentándose
Tabla 4. Relación entre presencia y ausencia de los síntomas y signos clínicos: fiebre (A), compromiso respiratorio (B), cefalea (C), síntomas gastrointestinales (D) en relación a serología IgM anti-hantavirus por método de referencia

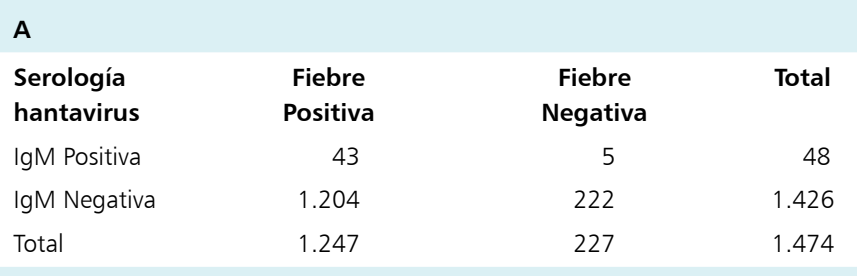

S: 90\%; E: 16\%; VPP: 3\%; VPN: 98\%, LR+: 1,57; LR-: 0,99.

B Serología Compromiso Compromiso Total hantavirus Respiratorio Positivo Respiratorio Negativo

$\begin{array}{lrrr}\text { IgM Positiva } & 21 & 21 & 42 \\ \text { IgM Negativa } & 391 & 856 & 1.247 \\ \text { Total } & 412 & 877 & 1.289\end{array}$

S: 50\%; E: 69\%; VPP: 5\%; VPN: 98\%, LR+: 2,13; LR-: 0,97.

C

\begin{tabular}{lccc}
$\begin{array}{l}\text { Serología } \\
\text { hantavirus }\end{array}$ & $\begin{array}{c}\text { Cefalea } \\
\text { Positiva }\end{array}$ & $\begin{array}{c}\text { Cefalea } \\
\text { Negativa }\end{array}$ & Total \\
IgM Positiva & 40 & 11 & 51 \\
IgM Negativa & 1.136 & 240 & 1.376 \\
Total & 1.176 & 251 & 1.427 \\
\hline
\end{tabular}

S: 78\%; E: 17\%; VPP: 3\%; VPN: 96\%, LR+: 0,78; LR-: 1,01

D

$\begin{array}{lccr}\begin{array}{l}\text { Serología } \\ \text { hantavirus }\end{array} & \begin{array}{c}\text { Síntoma GI } \\ \text { Positivo }\end{array} & \begin{array}{c}\text { Síntoma Gl } \\ \text { Negativo }\end{array} & \text { Total } \\ \text { IgM Positiva } & 29 & 17 & 46 \\ \text { IgM Negativa } & 808 & 534 & 1.342 \\ \text { Total } & 837 & 551 & 1.388\end{array}$

S: $63 \% ;$ E: 40\%; VPP: 3\%; VPN: 97\%, LR+: 1,12; LR-: 1,00

en nuestro país en forma endémica con un marcado aumento estacional (noviembre a abril). Hasta el año 2015 se ha notificado un total de 963 casos (www.epi.minsal.cl), con una mortalidad que fluctúa entre $30 \mathrm{y}$ $40 \%$. Para el médico clínico, la sospecha diagnostica constituye un desafío por la inespecificidad de los síntomas prodrómicos. Estos síntomas incluyen fiebre, cefalea, mialgias y síntomas gastrointestinales, los que preceden a los síntomas respiratorios y se asemejan a los síntomas de otras enfermedades de etiología infecciosa ${ }^{12}$.

En el presente análisis, se evaluó la $\mathrm{S}$ y E de los signos y síntomas definidos para los casos de sospecha de enfermedad por hantavirus. Una limitante en este análisis fue que $58,6 \%$ de los formularios de notificación de caso sospechoso de infección por hantavirus fueron 
incompletos. De los parámetros de laboratorio, cerca de $50 \%$ de las notificaciones carecían de registro (Tabla 2). Lo anterior demuestra la falta de adhesión a la definición de caso clínico sospechoso del Ministerio de Salud, donde se establece que:

Se debe sospechar infección por hantavirus cuando existe uno o más antecedentes de situaciones de riesgo epidemiológico de exposición al agente dentro de las seis semanas previas al inicio de los síntomas y la presencia de, a lo menos, dos de los siguientes signos y sintomas: fiebre, cefalea y mialgias, con o sin sintomas gastrointestinales, presencia de inmunoblastos (linfocitos atípicos) $>10 \%$, recuento de leucocitos con desviación a izquierda y/o hemoconcentración, radiografía de tórax normal o con infiltrado intersticial uni o bilateral (Guía MINSAL, www.minsal.cl) ${ }^{9}$.

Los síntomas y signos prodrómicos consignados con mayor frecuencia fueron fiebre, cefalea, mialgias y síntomas gastrointestinales.

Interesantemente, los datos muestran que la presencia de fiebre como criterio de sospecha de infección hantavirus presenta una S de $90 \%$ y E de $16 \%$, con VPP de $3 \%$, dificultando la utilización de este criterio como signo único de asociación con la presencia de la enfermedad. Sin embargo, el VPN del parámetro fiebre es de $98 \%$, lo cual sí representa una herramienta útil para descartar la asociación con la enfermedad.

Considerando los antecedentes expuestos, y la ausencia de fiebre en $9,4 \%$ de los casos confirmados positivos para enfermedad por hantavirus, es de suma importancia incorporar en el informe de notificación la temperatura en grados Celsius (como parámetro cuantificable), y aportar el antecedente de haber recibido o no antipiréticos, con el fin de establecer un punto de corte que discrimine sospecha.

Al analizar los parámetros del hemograma de manera individual, se aprecia que la trombocitopenia presenta la mayor S para sospecha de infección por hantavirus, encontrándose presente en $98 \%$ de los pacientes con SCPH confirmado.

El hallazgo clínico con mayor E fue la presencia de inmunoblastos (>10\%) con un VPP de 98\%. La presencia de estos dos parámetros, trombocitopenia y recuento de inmunoblastos (>10\%) son elementos de gran importancia para la sospecha clínica adecuada, y el diagnóstico precoz del SCPH. Si bien el parámetro hemoconcentración presenta una E de 98\%, su VPP es sólo de 18\%, parámetro que según series clínicas va acentuándose con la progresión de la enfermedad ${ }^{14,15}$.

En nuestro estudio el VPN de trombocitopenia fue de $100 \%$, lo que refleja que la ausencia de este parámetro se asocia con una menor probabilidad de presentar la enfermedad. De lo anterior, se hace de especial importancia para el médico clínico observar la progresión de la trombocitopenia en el desarrollo de la enfermedad, como parámetro indicador de sospecha, más que un valor único. Esto al considerar que el VPP fue de $16 \%$ en esta serie. En relación a la presencia de inmunoblastos ( $>10 \%)$ el VPP fue de $37 \%$, lo que resalta la importancia de capacitar al personal de laboratorio en la lectura de este parámetro.

Considerando que un cociente de probabilidad (LR) positivo $>10$ y un cociente de probabilidad negativo $<$ 0,1 indican generalmente que un ensayo tiene méritos suficientes para ser una herramienta de diagnóstico útil ${ }^{13}$. La evaluación de LR reafirma que el parámetro inmunoblastos presentó mejor capacidad para la sospecha adecuada de pacientes infectados con hantavirus (LR+12,1). Por otra parte, el parámetro trombocitopenia presentó la mejor capacidad para garantizar la confianza de una baja probabilidad de infección por hantavirus (LR- 0,03).

Dada la necesidad de contar con una definición de caso sospechoso de máxima $\mathrm{S}$ que permitiera establecer la sospecha de infección por hantavirus de manera precoz, la Universidad de Nuevo México realizó un análisis de asociación de parámetros clínicos y de laboratorio con el fin de generar un triage de los casos sospechosos de infección. En dicho estudio se implementó un tamizaje de frotis de sangre periférica, basado en cinco criterios; trombocitopenia, presencia de desviación izquierda en los leucocitos, hemoconcentración, ausencia de granulaciones patológicas y la presencia de inmunoblastos. En el momento de la implementación, el tamizaje de frotis de sangre periférica tenía una sensibilidad de $96 \%$ con la presencia de cuatro de cinco parámetros, con E de $99 \%$, un VPP de $83 \%$, y VPN de $99 \%{ }^{14,16}$.

En nuestro estudio no fue posible establecer relación de número de parámetros alterados versus resultados de serología, ya que en las notificaciones de casos sospechosos, los datos fueron consignados de manera deficiente. Eso explica que, de los 53 casos confirmados positivos para infección por hantavirus, sólo 24 (45,3\%) presentaran la información de tres parámetros (hemoconcentración, trombocitopenia e inmunoblastos). De los 1.513 casos confirmados como negativos para infección por hantavirus, sólo $432(28,6 \%)$ presentaban la información de estos tres parámetros.

Llama la atención la elevada sospecha de enfermedad por hantavirus versus el bajo porcentaje de casos confirmados $(3,4 \%)$. Esto podría reflejar la falta de disponibilidad de laboratorio las $24 \mathrm{~h}$ en algunas regiones, falta de entrenamiento en la lectura de inmunoblastos en el hemograma y la ocurrencia de casos clínicos con resultado fatal; situaciones que conllevan a un aumento de la sospecha clínica por los médicos tratantes, muchas veces alejados de hospitales de referencia.

En base a lo descrito anteriormente, es necesario reforzar la definición de caso sospechoso y proponer un algoritmo que complemente la propuesta actual. 
Proponemos reforzar la definición de caso sospechoso acentuando la necesidad de evaluar en forma obligatoria tres parámetros: fiebre, trombocitopenia $\left(<150.000 / \mathrm{mm}^{3}\right)$ e inmunoblastos $(>10 \%)$ en el hemograma.

Además, en aquellos casos en que el antecedente epidemiológico de sospecha de infección por hantavirus sea alto y asociado a presencia de fiebre sin trombocitopenia, se sugiere realizar un segundo hemograma dentro de 8-12 h con el fin de evaluar la evolución del recuento plaquetarío, ya que éste es uno de los signos de presentación precoz en la enfermedad que presenta un deterioro progresivo ${ }^{14,17}$.

En resumen, se propone la discusión de la siguiente definición de caso sospechoso de infección por hantavirus: "si existe uno o más antecedentes de situaciones de riesgo epidemiológico de exposición al agente dentro de las seis semanas previas al inicio de los sintomas y la presencia de tres o más de los siguientes signos y sintomas, incluyendo los destacados fiebre, cefalea y mialgias, con o sin sintomas gastrointestinales, presencia de trombocitopenia $<150.000 / \mathrm{mm}^{3}$, inmunoblastos (linfocitos atípicos) $>10 \%$, recuento de leucocitos con desviación a izquierda y/o hemoconcentración, radiografía de tórax normal o con infiltrado intersticial uni o bilateral". Siendo obligatoria la presencia de fiebre y trombocitopenia (Guía MINSAL, www.minsal.cl) ${ }^{9}$.

En aquellos casos ajustados a la definición se sugiere realizar estudio con técnicas rápidas que detecten $\operatorname{IgM}$ anti-hantavirus ${ }^{17,18}$. Según datos preliminares del laboratorio (no publicados aún) el ensayo inmunocromatográfico IgM POC Puumala mostró sensibilidad y especificidad de $97 \%{ }^{10,17}$.

Finalmente, se hace necesario reiterar a nivel del sistema sanitario chileno la importancia de contar con datos completos en los formularios de notificación, evaluar la red de laboratorios y su capacidad de realizar hemograma de urgencia e implementar programa de capacitación para lectura de inmunoblastos en la red de hospitales.

Agradecimientos. Este trabajo forma parte de la temática desarrollada por el Proyecto FIC15-20 IDI 30421494 financiado con recursos del Fondo de Innovación para la
Competitividad del Gobierno Regional de Los Ríos y la Universidad Austral de Chile. Convenio Hantavirus Servicio de Salud Valdivia y Universidad Austral de Chile. DID-UACH.

\section{Resumen}

Antecedentes: Actualmente en Chile, debido a la elevada sospecha clínica de enfermedad por hantavirus y el alto impacto en salud pública que esto provoca, se hace necesario reforzar al equipo de salud, los criterios de sospecha clínica y epidemiológica de hantavirosis. Objetivo: Analizar la información contenida en las notificaciones de sospecha de infección por hantavirus versus la técnica de referencia para el diagnóstico confirmatorio de casos sospechosos, ELISA IgM de captura anti-hantavirus. Material y Método: Mediante cálculo de precisión diagnóstica se analizó la correlación que existe entre la información entregada en las notificaciones versus el resultado de la confirmación mediante la técnica de referencia. Resultados: De 1.566 pacientes estudiados $3,4 \%$ (53 casos) fue confirmado para SCPH. De las notificaciones analizadas $58,6 \%$ estaban con datos incompletos. Los porcentajes de positividad de la técnica de referencia asociada a fiebre, mialgia y cefalea, fueron de $80-85 \%$. Destaca que la presencia de inmunoblastos $(>10 \%)$, presenta: S: 25\%, E: 98\%, VPP: 37\%, VPN: 97\%. Para trombocitopenia se obtuvo: S: 98\%, E: 74\%, VPP: 16\%, VPN: 100\%. Conclusión: Se hace necesario reiterar a nivel del sistema sanitario chileno la importancia de contar con datos completos en los formularios de notificación. La presencia de trombocitopenia e inmunoblastos $(>10 \%)$ fue altamente sensible y especifica, respectivamente, en la detección de pacientes con SCPH. Con el fin de optimizar la sospecha de infección por hantavirus, según la definición de caso sospechoso, se plantea la necesidad de desarrollar programas de capacitación para la sospecha clínica y lectura de parámetros de laboratorio, tales como presencia de inmunoblastos en el hemograma, así como incluir un algoritmo con el fin de optimizar la sospecha y el uso adecuado de los recursos sanitarios.

\section{Referencias bibliográficas}

1.- Navarrete M, Mansilla R, Saldías F, Ferrés M, Zaror L. Investigación retrospectiva de casos de síndrome pulmonar por hantavirus en Valdivia, 1993. EPI Visión 1998; 19: 3.

2.- Baró M, Vergara J, Navarrete M. Hantavirus en Chile: revisión y análisis de casos desde 1975. Rev Med Chile 1999; 127: 1513-23.

3.- López N, Padula P, Rossi C, Miguel S, Edelstein A, Ramírez E, et al. Genetic characterization and phylogeny of Andes virus and variants from Argentina and Chile. Virus Res 1997; 50: 77-84.

4.- Medina R A, Torres-Péez F, Galeno H, Navarrete M, Vial P, Palma R E, et al. Ecology, genetic diversity, and phylogeographic structure of Andes virus in humans and rodents in Chile. J Virol 2009; 83: 2446-59.

5.- Schmaljohn C, Hjelle B. Hantaviruses: A global diseases problem. Emerg Infect Dis 1997; 3: 95-104.

6.- Botten J, Mirowsky K, Ye C Y, Gottlieb K,
Saavedra M, Ponce L, Hjelle B. Shedding and intracage transmission of Sin Nombre hantavirus in the deer mouse (Peromyscus maniculatus) model. J Virol 2002; 76: 7587-94.

7.- Padula $P$, Figueroa R, Navarrete $M$, Pizarro E, Cadiz R, Bellomo C, et al. Transmission study of Andes hantavirus infection in wild sigmodontine rodents. J Virol 2004; 78: 11972-9.

8.- Sotomayor V, Olea A, Labraña M. Diagnostic and treatment of cardiopulmonary syndrome. 
Chile-2007. Rev Chilena Infectol 2009; 26: 68-86.

9.- Guía clínica de prevención, diagnóstico y tratamiento del SCPH http://web.minsal.cl/ sites/default/files/files/HANTA_imprimir.pdf. 27/012016.

10.- Navarrete M, Barrera C, Zaror L, Otth C. Rapid immunochromatographic test for hantavirus andes contrasted with capture-IgM ELISA for detection of Andes-specific IgM antibodies. J Med Virol 2007; 79: 41-4.

11.- Padula P J, Rossi C M, Della M O, Martínez P V, Colavecchia S B, Edelstein A, et al. Development and evaluation of a solidphase enzyme immunoassay based on Andes hantavirus recombinant nucleoprotein. J Med Microbiol 2000; 49: 149-55.
12.- Hinojosa M P, Villagra E C, Mora J R, Maier L. Identificación de otros agentes infecciosos en casos sospechosos de síndrome cardiopulmonar por hantavirus. Rev Med Chile 2006; 134: 332-8.

13.- Jaeschke R, Guyatt G, Sackett D. Users' Guide to the Medical Literature. III. How to use an article about a diagnostic test. B. What are the results and will they help me in caring for my patients? The Evidence-Based Medicine Working Group. J Am Med Assoc 1994; 271: 703-7.

14.- Koster F, Foucar K, Hjelle B, Scott A, Chong Y Y, Larson R, et al. Rapid presumptive diagnosis of hantavirus cardiopulmonary syndrome by peripheral blood smear review. Am J Clin Pathol 2001; 116: 665-72.

15.- Knust B, MacNeil A, Rolli P E. Hantavirus pulmonary syndrome clinical findings. Vector Borne Zoonotic Dis 2012; 12: 393-439.

16.- Dvorscak L, Czuchlewski D R. Successful triage of suspected hantavirus cardiopulmonary syndrome by peripheral blood smear review a decade of experience in an endemic region. Am J Clin Pathol 2014; 142: 196-201.

17.- Riquelme R, Rioseco M L, Bastidas L, Trincado L, Riquelme M, Loyola H, et al. Hantavirus pulmonary syndrome, Southern Chile, 1995-2012. Emerg Infect Dis 2015; 21: 562-8.

18.- Latus J, Schwab M, Tacconelli E, Pieper F M, Wegener D, Rettenmaier B, et al. Acute kidney injury and tools for risk-stratification in 456 patients with hantavirus-induced nephropathia epidemica. Nephrol Dial Transplant 2015; 30: 245-51. 